\title{
XXXIV.
}

(Aus der Pflegeanstalt Rheinau: Direktor Dr. Ris.)

\section{Zur Kasuistik \\ der juvenilen Form der amaurotischen Idiotie, mit histopathologischem Befund.}

\author{
Von \\ Dr. T. Rogalski, \\ Assistenzarzt.
}

(Hierzu Tafel X̣VIII.)

Das typische Bild der Tay-Sachsschen Krankheit (infantiler Form der amaurotisch-familiären Idiotie) ist klinisch, wie anatomopathologisch durch Arbeiten von Falkenheim, Sachs, Apert, Schaffer und anderen so gut abgegrenzt, dass die Mitteilung eines vereinzelten typischen Falles kaum eine lohnende Aufgabe wäre. Anders steht die Sache mit der juvenilen Form dieser Erkrankung. 1896 weist Higier1) auf die Aehnlichkeit seiner unter dem Namen der familiären spasmodischen Diplegie mit Amaurose beschriebenen Fälle mit der Tay-Sachsschen Krankheit hin. In zusammenfassenden Arbeiten teilt dann $\operatorname{Vog}^{2}{ }^{2}$ ) weitere in der Jugendzeit erkrankten Fälle mit, zieht eine genaue Parallele zwischen den in den ersten Lebensjahren beginnenden und der im späteren Alter auftretenden Krankheit und kommt zum Schlusse, es handle sich um zwei verschiedene Formeu derselben Erkrankung. Sehaffer ${ }^{3}$ ), der diesen Erkrankungsprozess am ausführ-

1) H. Higier, Ueber die seltenen Formen der hereditären und familiären Hirn- und Rückenmarkskrankheiten. Deutsche Zeitschr. f. Nervenheilkunde. Bd. IX. 1896.

2) H. Vogt, Ueber familiäre amaurotische Idiotie and verwandte Krankheitsbilder. Monatsschr. f. Psych. u. Neurologie. Bd. XVIII. 1905. H. Vogt, Zur Pathologie und pathologischen Anatomie der verschiedenen Idiotieformen. Monatsschr. f. Psych. u. Neurologie. Bd. XXII. 1907.

3) K. Schaffer, Ueber die Anatomie und Klinik der Tay-Sachsschen amaurotisch-familiären Idiotie mit Rücksicht auf verwandte Formen. Zeitschr. f. d. Erforsch. u. Behandl. d. jugendl. Schwachsinns. Bd.III. 1909. Sonderabdr. 
lichsten bearbeitet hat, äussert sich ïber die beiden Formen in seiner letzten Arbeit folgendermassen: „... die schwerere, rasch verlaufende Sachssche Form lässt sich mit der leichteren, langsam rerlaufenden Spielmeyer-Vogtschen Form in eine anatomisch identische, scharf gekennzeichnete, einheitliche grosse klinische Familie, welche wir die amaurotisch familiäre Idiotie nennen, zusammenfassen".

Ganz anderer Ansicht ist Apert ${ }^{1}$ ). Er meint, man soll die Fälle von Spätform für sich klassifizieren, weil sie mit der Tay-Sachsschen Krankbeit gar nichts zu tun haben. In der letzten Zeit unterscheidet auch Higier ${ }^{2}$ ) scharf die beiden Formen, die anatomo-pathologiseb ähnlich, klinisch in hohem Grade verschieden seien.

Die Ursache dieser verschiedenen Anschauungen scheint mir in dem Umstande zu liegen, dass wir gegenüber den - hier den Ausscblag gebenden - zahlreichen übereinstimmenden anatomischen Befunden der infantilen Form nur sehr wenige histologisch genauer untersuchten Fälle der Spätform besitzen. Es sind vor allem die ausführlich beschriebenen anatomischen Veränderungen von Spielmeyer ${ }^{3}$ ) und Vogt $\left.{ }^{4}\right)$. Und auch diese unterscheiden sich vicht nur quantitativ von den Fällen infantiler Form, sondern auch in gewissem Grade qualitativ unter sich.

Dieser Umstand veranlasst mich zur Veröffentlichnng des nachstehend geschilderten Falles der juvenilen Form. Obwohl ich nur das Gehirn zur Verfügung hatte und keine Fibrillenmethode anwenden konnte, scheint doch das Ergebnis der histologischen Untersuchung genügend zu sein zur Feststellung, dass dieser Fall in den anatomischen Veränderungen des Zentralnervensystems sehr nahe denen der TaySach sschen Krankheit steht.

Zu Lebzeiten der Patientin schwankte die Diagnose zwischen Idiotie mit Amaurose und juveniler Paralyse. Es war in der Zeit, wo man bei uns von der Tay-Sachsschen Krankheit recht wenig, und von der juvenilen Form dieser Erkrankung gar nichts wusste. Erst nach ein paar Jahren konnte Direktor Dr. Ris auf Grund der neueren Publikationen dem Fall den richtigen Platz zuweisen, nachdem er den anatomischen

1) Apert, Lidiotie amaurotique familiale (maladie de Tay-Sachs). La semaine médicale. 1908.

2) H. Higier, Zur Klassifikation der endogenen Hirnlähmungen (Diplegiae cerebrales). XVI. internat. mediz. Kongr. in Budapest. 1909. Autoreferat i. Neurol. Centralbl. 1909. Nr. 19.

3) W. Spielmeyer, Klinische und anatomische Untersuchungen über eine besondore Form von familiärer amaurotischer Idiotie. Habilitationsschrift. Gotha 1909.

4) H. Vogt, l. c. 
Befund wiederholt in Fachkreisen als einen unter seinem Material einzig dastehenden und rätselhaften demonstriert hatte, und auch von anderer Seite keine Aufklärung darüber hatte bekommen lzönnen. Er hat mir dieses Jahr sein Material zur Verfügung gestellt und ich danke Ihm bestens, dass er mich in den Stand setzte, die folgenden Untersuchungen zu machen.

Bertha P. stammt aus einer schweizerischen (von Alters her in Zürich ansässigen) Familie. Die Grosseltern starben an Alterssehwäche. Der Vater soll nie krank gewesen sein und sieht auch jetzt im 70. Lebensjahre noch recht gut aus. Die schwächlich und zart gebaute Mutter hatte einen Herzfehler und starb an Wassersucht im Alter von 33 Jahren. Von vier Kindern ist die älteste Tochter 38 Jahre alt, lebt, ist gesund und verheiratet. Das zweite Kind starb 14 Tage nach der Geburt, dann folgt unsere Kranke, 1875 geboren. Der jüngste Sohn, jetzt 32 Jahr alt, lebt, ist gesund und verheiratet. Von Kindern der Geschwister unserer Kranken soll keines auf ähnliche Weise wie sie erkrankt sein.

Patientin selbst soll sich trotz in früher Jngend überstandenen Scharlachs und Diphtherie bis zum 7. Lebensjahre vollständig normal entwickelt haben; der Umgebung ist nichts besonderes an ihr aufgefallen. Mit 7 Jahren ging sie in die Schule und besuchte sie durch sieben Jahre. Sie kam aber nur bis zur 4. Klasse, weil sie jede Klasse zweimal besuchen musste. Immerhin lernte sie schreiben und lesen. An Gang und Sprache hat man nie etwas abnormes beobachtet. Nachdem Patientin zwei Jahre die Schule besucht hatt, erkrankte sie an Typhus (grosse Typhus-Epidemie in Zürich 1883/1884). Sie genas dann, soll wieder völlig normal gewesen sein und besuchte weiter die Schule. Inzwischen starb ihre Mutter. Der Vater heiratete zum zweitenmal und es fiel bald der Stiefmutter auf, dass das Mädchen "kurzsichtig" sein müsse. In der Meinung, es werde ihm eine Brille verschrieben, wandte sie sich an Dr. Horner, den damaligen Professor der Augenheilliunde in Zürich.

Im Poliklinikprotokoll des Jahres 1885 findet sich unterm 8. VIII. notiert: "Rechts: Seben = Fingerzählung in 5 Metern; links: Sehen $=\mathrm{S} 1 / 10$. Papillen blass und undurchsichtig. Makula in ganzbestimmter Weise erkrankt: um eine hellrote ovale Figurgeht ein dichtergefranster Pigmentkranz herum, und dies auf beiden Augenganz gleich. Diagnose: Atrophia post nenrit. Macula-Affectio. Das Kind habe vor drei Jahren Typhus und Diphtherie durchgemacht, seitdem Sehvermögen nicht so gut wie früher. Intelligenz auch ziemlich stark herabgesetzt. Kind sehr klein, Schädel zusammengedrückt."

Nach ca. $3 / 4 \mathrm{Jahr}$ wurde wegen desselben Leidens Prof. Dr. O. Haab konsultiert. In seinen Protokollen findet sich unterm 28.IV.1886folgendes: „Sehen nicht gut, ging bis jetzt in die Sehule. Seit einigen Woohen wurde besondere Abnahme des Sehens bemerkt. Ophtalmoskopisch: Leichte Atrophie der Papille, die zugleich etwas verwaschen aussieht, als ob ein heller, weisser, dünner Schleier davor läge. Zugleich Arterien und Venen, bssonders aber 
erstere sehrschmal. Beiderseits Makula affektion und in derPeripherie, sobon ziemalich bald gegen dieselbe beginnend, feine, bräunliche Tüp felung a u grau-rotem Grund, in dem ausserdem ganz verwaschene, helle, rundliche Stellen aus der Tüpfelung sichtbar werden, sieht beiderseits blos Finger in nächster Nähe. Die Pupillen sind meist sehr weit."

Ich rerdanke auch hier Herrn Prof. Haab die gütige Mitteilung der beiden Protokolle.

Patientin besuchte dann noch mit Unterbrechung die Schule, bis die Erblindung vollständig war. Sie soll dann die Leute nach der Sprache richtig erkannt haben. Die Geistesstörung machte sich auch immer mebr bemerkbar, besonders in Form von Gedächtnisschwäche. Im Alter von ca. 13 Jahren traten weitere Störungen anf, nänlich am Gang und an der Sprache, und mit dem Eintreten der Menses im 14.-15. Jahre bekam die Kranke zum erstenmal epileptiformen Anfall. Weitere traten ungefähr alle vier Wochen auf, 3 bis 4 Tage nacheinander je ein Anfall.

In der Zeit jwischen dem 14.-23. Lebensjahre finden wir das allmähliche Fortschreiten aller erwähnten Störungen. Die Sprache wurde scbwieriger, bis die Kranke, die früher alle Fragen richtig beantwortet haben soll, nur nooh "Vater", "Matter", "Ja" oder "Nein" rief; sonst sei nichts verständlich gewesen. Psychisch ist sie ganz teilnahmlos und blödsinnig geworden. Den epileptiformen Anfall bekam sie jetzt jede Woohe. "Wenn derselbe nicht auftrat, dann sei ihr geistiger Zustand noch schlechter gewesen. Beim Anfall fiel sie stark hin, bekam Schaum vor dem Mund, oft sei derselbe blutig, bekam Krämpfe und wurde starr. Vor den Anfällen war sie immer stark aufgeregt, nach den Anfällen lag sie zwei Stunden lang bewusstlos, schlief dann viel und nahm gar keine Nahrang zu sich. Auch sonst machten oft die Nervenzuckungen mit Kopf und Händen notwendig, dass man ihr za essen gab, wie einem Kleinen. Sie wurde dick und schwer." (Brief der Stiefmutter.)

Die Kranke wurde zuerst 1/2 Jahr von einem Arzt mit Thyreoidtabletten, dann $21 / 2$ Jahr von eíner Kurpfuscherin mit Bauscheidtismus behandelt alles ohne Erfolg. Die Menstruation wurde unregelmässig und ist schliesslich ausgeblieben.

Aus dem am 25. 3. 1898 behufs der Aufnahme in die Anstalt ausgestellten Zeugnis erfahren wir folgendes über den Zustand unserer damals 23 Jahre alten Kranken: Absolute Teilnahmslosigkeit, vollständige Lähmung der Extremitäten, Sensibilität soheint herabgesetzt zu sein, Pupillenstarre, völlige Blindheit, Sprachlosigkeit, Unreinlichkeit (Patientin lässt Stahl und Urin unter sich), sehr unregelmässige Stuhlentleerungen. Appetit gat, doch nicht auffallend stark. Die Haut ist mit den rom Bauscheidtismus herrührenden Narben bedeckt. 28. Juni 1898 wurde die Kranke in die Pflegeanstalt Rheinau aufgenommen. Die Untersuchung ergab folgende Resultate:

Körperlicher Befund: Kleine Person von gutem knochen-und mässigem Muskelbau. Paniculus adiposus ziemlich entwickelt. An derHaut des Rumpfes befinden sich eigenartige Veränderungen in Form von $1 \mathrm{~cm}$ langen und $2-3 \mathrm{~cm}$ breiten milchweissen Flecken. Die Haut scheint an diesen Stellen schlaffer zu sein, 
als an den normalen Partien, welche dazwischen liegen (Bauscheidtnarben). Kopfumfang $51 \mathrm{~cm}$; Processus occipitalis-Glabella $17 \mathrm{~cm}$, grösste Breite $15 \mathrm{~cm}$. Gesicht gedunsen, Wangen stark entwickelt, Stirn schmal and niedrig, Mund gross, Lippen wulstig, Zähne ziemlich gut erhalten, kurz; Schneidezähne zeigen abgerundete Enden (hered. Lues?). Pupilleu eng, Strabismus convergens. Von Zeit zu Zeit Konvulsionen der Augenmuskeln (Nystagmus) und an den oberen Extremitäten. Innere Organe ohne krankbafte Veränderungen.

Wegen der Unruhe der Patientin war die Untersuchung des Augenhintergrundes sehr erschwert. Weder das Bild der Papille noch der Macula lutea kounte man erhalten. Nach vielen staubförmigen Pigmentationen, die sich mehr auf der Peripherie der Retina bemerkbar machten, hat man die Diagnose auf Retinitis pigmentosa gestellt.

Psychischer Befund: Die im Bett bleibende Patientin gab zuerst gar keinenLaut von sich. Erst nach einigenTagen liess sie aufFragen ein fastunverständliches " Ja" oder "Nein" hören. Patientin hat die Augen in wachem Zustande meist offen in fast beständiger Unruhe, reagiert wenig auf Veränderung in ihrer Umgebung; bebält dabei immer gleiche Haltung. Der stupide idiotenhafte Gesichtsausdruck bleibt fast ohne Veränderung. In wie weit das Gehör vorbanden, ist nicht sicher festzustellen: wird die Kranke in gewöhnlicher Weise angesprochen, so zeigt sie gar keine Reaktion darauf; erst wenn man sie in nächster Nähe anruft, scheint sie zu reagieren. Sie öffnet dann die Augen mehr, kewegt den Kopf usw., fängt auch $a b$ und zu an zu lachen, wenn sie angerufen wird. Begrüssung mit der Hand erwidert sie bie und da auf dieselbe Weise. Sonst aber gibt sie keine Auskunft. In der Nacht ruhig, lässt Urin und Stuhl unter sich gehen. Beständige Verstopfung wird durch Klysmen beseitigt. Die Kranke kann nicht selbständig essen; wenn ihr aber das Essen in den Mund gereicht wird, isst sie mit ziemlicher Gier.

Durch lange Zeit hat man nur einen epileptischen Anfall gesehen. Später waren die Anfälle häufiger, die Kranke war vor ihnen gereizt, warf alles weg, stiess die Hand von sich, machte böses Gesicht und weinte. Sonst war sie fröhlich, hatte es gern, wenn man sich mit ihr abgab, lachte, aber sprach nichts. Ausser diesen spärlichen Zeichen des Geisteslebens war die Patientin ganz verblödet. In Tragbahren in die Anstalt gebracht, kounte sie dann noch längere Zeit mit Hilfe der Wärterin gehen.

Nach fünf Monaten des Aufenthaltes soll die Patientin - nach Aussage derWärterin - spontan und dentlich gesagt haben: „Spitzliwie“ (statt „Spitzwiesli" = Krankengarten, in dem die Kranke oft verweilte). Später hat sie nie ein Wort gesprochen.

1899 wurden die Anfälle häufiger, die Patientin wurde apathisch und völlig teilnahmslos. Jede Berührung löste Zusammenschrecken und allgemeine Zuckungen aus. Die Entwicklung einer Cataracta complicata erlaubte nicht bei der jetzt ruhigeren Patientin einen Augenhintergrund zu erhalten.

1900 nahm die vollkommen verblödete Kranke rapid ab. Es traten Kontrakturen der unteren Extremitäten (Foetalhaltung) und an den Füssen Pemphigusblasen auf, die zu schlecht heilenden Wunden fübrten. 
1901 entstanden wiederholt Pemphigusblasen. Trotz Wasserkissen, Luftringen und Dauerbädern war ausgedehnter Decubitus äber dem Kreuzbein und beiden Trochanteren nicht zu verhindern. Der Kräfteverfall nabm stark zu. 20. und 21. Juni kam je ein epileptischer Anfall. Seither konnte der Kranken gar keine Nahrung beigebracht werden. Am nächsten Tag (22. 4.) starb sie im allgemeinen hochgradigen Marasmus. Unmittelbar vor dem Tode war sie sehr cyanotisch.

Die sieben Stunden nach dem Tode der Kranken vorgenommene Sektion des Gehirns ergab folgende Resultate:

Schädeldach lässt sich nur im Zusammenhang mit der Dura abheben. Dasselbe ist schwer und dick, etwa assymmetrisch mit Erweiterung nach links hinten; Diploegehalt gering. Dura entlang der Sagittalnaht fest adhärierend, auf der Innenfläche, besonders links, zahlreiche, stecknadelkopfgrosse zum Teil konfluierende, gelbbraune, nicht ahwischbare Auflagerungen (Pachionische Granulationen). In der hinteren Schädelgrube etwa $1 \mathrm{ccm}$ klare, etwas blutig tingierte Flüssigkeit. Sinus der Basis leer. Gefässe der Gehirnbasis zartwandig, mit wenig verschieblichem Blut. Pia wenig bluthaltig, wenig ödematös, wenig verdickt, löst sich leicht.

Gehirnwindungen sämtlich sehr stark verschmälert, klaffen aber nicht; ihre Oberfläche erscheint gerunzelt, punktiert, zernagt. Hirnsubstanz zähe und derb. In den Ventrikeln nur wenig klare Flüssigkeit. Beide Tractus olfactorii sehr atrophisch, beinahe fadenförmig, von grau durchscheinender Farbe. Im Ventriculus IV Andeutung feiner Granulationen. Rinde gegen die weisse Substanz stark abgesetzt, speckig durchscheinend, aber nicht hochgradig verschmälert. Gesamtgewicht des Gehirns (ohne Häute und Liquor) $=670 \mathrm{~g}$.

Einzelne Stückchen derRinde werden teils in Sublimat, teils in Formol gehärtet und in Paraffin eingebettet. Das ganze Gehirn in Kalium bichromi$\mathrm{c} u \mathrm{~m}$ eingelegt.

Die Leiche von infantilem Körperbau war extrem abgemagert, mit bochgradigen Flexionskontrakturen des Hüft- und Kniegelenkes. An den oberen Extremitäten weniger starke Kontrakturen vorhanden. Ueber Kreuzbein, Trochanter, Spina und Crista iliaca und Olecaranon tiefgehender Decubitus, Totenstarre wenig ausgesprochen.

Die übrigen Organe wurden im Pathologischen Institut in Zürich seziert. Im Sektionsprotokoll findet man ausser einfacher starker Atropbie der Muskulatur und geringer Atrophie der Leber und Milz, noch als wichtigsten Befund: Pleuritis fibrinosa sinistra über den Oberlappen, Bronchitis purulenta, Bronchopneumonie, Emphysem und geringes Lungenödem.

Mikroskopischer Befund: Das mir überlassene anatomische Material bestand aus den in Paraffin eingebetteten Hirnrindenstückchen und dem seit 1901 in Kalium bichromicum liegenden Gehirn. Die Auswahl der Methoden war daher recht gering und ich musste von vorn berein auf die Fibrillenbilder verzichten. Die Sublimatfixierung der Hirnrindestückehen für die Methylenund Toluidinblaufärbung, wie sie bei diesem Fall noch angewandt 
ist, wurde in späterer Zeit zu Gunsten der Alkoholfixierung verlassen. Nach vielfachen Vergleichen ergab sich, dass die Sublimatbehandlung. in Bezug auf Formerhaltung und Färbbarkeit der Elemente der Hirnrinde keine nennenswerten Unterschiede gegen die Alkoholbehandlung ergibt. Die nachfolgend beschriebenen Resultate dürfen also ohne Weiteres mit an Alkoholmaterial erhaltenen Befunden verglichen werden.

Schon das Uebersichtsbild eines nach der Misslschen Methode gefärbten Präparates der Grosshirnrinde irgend einer Windung zeigt uns, dass wil es mit keinem normalen Gehirn zu tun haben. Es fällt nämlich sofort auf, dass bei annäbernd normalem Schichten-Typus und radiärer Stellung der Pyramidenzellen die Zellen selbst ungewöhnlich gross und in ihrer Form verändert sind (Fig. 1). Diese Störung, ein Schwellungsprozess eigentümlicher Art, betrifft fast sämtliche Zellen. Es ist fast unmöglich, eine normale Zelle zu finden. Die sternförmige Form der kleinen Zellen, mit ihren konkaven Wänden, sowie die grazile, eckige Form der kleineren und grösseren Pyramiden ist verloren gegangen. Die Zellen sind kugelig, bïnenförmig oder ganz unregelmässig aufgebläht. Die Nisslschen Schollen sind fast verschwunden, das Protoplasma sieht aus wie valuolisiert, der Kern ist dunkler gefärbt und meistens verlagert, das Kernkörperchen an die Wand gedrückt. Die Zellfortsätze kann man ziemlich weit verfolgen. Weiter bemerken wir das ungewöhnliche Verhalten des mittleren (manchmal auch eines seitlichen) Basalfortsatzes der Zelle: er ist weiter als gewöhnlich bei der Methylenblaufärbung sichtbar and zeigt eine isolierte Aufschwellung.

Ausser der Form der Zellen lenkt unsere Aufmerksamkeit sich auf runde, längliche oder ovale Lücken im Gewebe, die entweder perivaskuläre Räume bilden oder sich in der Nachbarschaft der Zellen befinden und dann auf ibre Form einen Einfluss ausüben. Im Innern oder im Mittelpunkt soloher Lücken liegt manchmal ein Neurogliakern. Noch besser als bei der Methylenblaufärbung (Fjg. 1) werden diese Gewebslücken sichtbar an mit Haemalaun gefärbten Präparaten von in Formol fixiertem Material (Fig. 2).

Um genauer die Art und den Grad der Erkrankung der Zellen kennen zu lernen, gehen wir zu Immersionsbildern über. Die polymorphen und spindelförmigen Zellen und die kleinen Pyramiden irgend einer Windung (Fig 3 , a bis d) oder die Sternzellen der Sehrinde zeigen uns die Verhältnisse am deutlichsten:

Der Leib der aufgeblähten Zelle besteht aus hellen Blasen und sein optischer Durchschnitt stellt den einer Wabe oder eines Netzes mit kleineren und grösseren Maschen dar. Dünne, rotblau gefärbte Züge der färbbaren Substanz mit etwas dickeren und dunkleren Knotenpunkten bilden ihre Wände. Der mässig grosse, ziemlich gleichmässig in einem blauen Ton gefärbte Kern von rundlicher oder ovaler Form rückt meistens an die Zellwand. Die Kernmembran ist deutlich erkennbar, das Kernkörperchen intensiv dunkelblau gefärbt und exzentrisch gelagert. - Bei noch weiter fortgeschrittener Erkrankung besteht der noch mehr und unregelmässiger aufgeblähte Zellleib (Fig. 3d) aus einigen grossen Blasen und ein paar kleineren 
Bläschen. Im Kern treten hellere Lücken hervor; die Kernmembran wird gefaltet und sieht aus, als wenn sie von angrenzenden Maschenwänden gebildet wäre. Oft ist der Kern an einer Stelle wie angefressen. Es kommen auch im Protoplasma eine oder mehrere das Jieht stärker brechende and gelblich aussehende Vacuolen zum Vorschein. -- Schlieslich trifft man Zellen, die den äussersten Grad der Erkrankung darstellen könnten: Die Wände der grossen Blasen verschwinden, die Fortsätze bröekeln ab, ans dem Kern bleiben nur einige, durch bläuliche Färbung erkennbare Reste and das resistente dunkle Kernkörperchen. Manchmal lässt uns die Gruppierung einzelner Bröckel und Körner die zu Grunde gegangene Zelle erkennen.

Ich nahm als Beispiel die kleineren, wenig Nissl-Substanz enthaltenden Zellen, weil man an ihnen die Art des Erkrankungsprozesses am besten beobachten kann. Dio Veränderung, welche sich an grösseren und grössten Pyramidenzellen kundgibt, ist nicht wesentlich von der oben geschilderten verschieden. Es überzeugen uns davon die in Fig. 4,5 und 6 wiedergegebenen Bilder.

Auch besteht hier das wichtigste Merkmal der Erkrankung darin, dass die Zellen stark geschwollen sind. Durch diese Schwellung haben sie ihre eckige Gestalt verloren und eine kugelige, öfters eine birnenförmge angenommen. Oft begegnet man auch einer starken Aufblähung der Seitenwand, die die Zelle ganz unregelmässig verunstaltet (Fig. 6). Der metachromatisch gefärbte Kern rückt meistens an die Abgangsstelle eines Dendriten oder an die Seitenwand. Bei birnförmig geschwollenen nimmt der kleine Kern eine kapuzenartige Gestalt an und wird durch die die Zelle auftreibende Kraft in den A pikaldendrit eingepresst. Das ziemlich grosse, dunkle Kernkörperchen liegt exzentrisch.

Was die Struktur des Protoplasma anbetrifft, so besteht der Unterschied von der bei polymorphen Zellen beschriebenen Veränderung nur darin, dass die polygonalen oder rundlichen Maschen des Netzes enger sind. Von den Nisslschen Schollen ist fast nichts mehr zu sehen. Nur in den wenig zahlreichen Zellen mit der Iokalisierten Aufblähung (Fig. 6) sieht man in dem weniger veränderten Teil, in welchem der Kern liegt, deutlich die Nisslschen Schollen. - Das sich sonst nicht färbende Hyaloplasma weist oft einen gelbbräunlichen Ton auf, der mehr oder weniger intensiv ist.

Abweichend von dieser allgemeinen Art der Frkrankung der Zellen stellen sich viele der tieferen grossen Pyramiden der vorderen Zentralwindung dar. Statt mit Schwellung haben wir es hier mit Schrumpfung zu tun. Die Zellen sind kleiner geworden, wie zusammengepresst, von einer länglichen, oft gebogenen Gestalt. Der ganze Zellinhalt ist intensiv dunkelblau gefärbt, und auf diesem Hintergrunde lässt sich noch eine in violettem Ton tingierte wabige Struktur erkennen, die von groben, dunklen Sohollen teilweise verdeckt ist. Die Stelle, wo der Kern liegt, macht sich meistens nur durch eine etwas heller blaue Färbung um das dunkle Körperchen bemerkbar. Die weit verfolgbaren, geschlängelten Seitendendrite nund der propfenzieherartig gewundene Apikaldendrit zeigen an den Dichotomie- und den Tmbiegungsstellen dieselben dunklen Schollen. 
Man begegnet auch Zellen, die den Eindruck der Uebergangsformen zwischen den letztgenannten und den früher geschilderten machen.

Gehen wir nun zu dem Verhalten der Fortsätze der geschwollenen Zellen über. Wie schon erwähnt wurde, fällt vor allem die isolierte Aufblähung des mittleren, manchmal auch eines seitlichen Basalfortsatzes ins Auge. - Die Stelle des Abganges dieses Fortsatzes von der Pyramidenzelle, der radiäre Verlauf und das Fehlen von dichotomischen Verzweigungen spricht dafür, dass es sich um den Axenzylinderfortsatz handelt. - Die Aufblähung zeigt eine ganz ähnliche netzartige Struktur, wie wir sie in den Zellen gesehen haben. Nur färbt sich das Netz hier sehr blass. Von der Zelle ist diese Auftreibung durch einen schmalen, manchmal etwas gesehlängelten, normal aussehenden und verschieden langen Teil des Fortsatzes getrennt. Nicht selten ist dieser Teil auch mehr oder wenig erweitert und der Zellinhalt geht kontinuierlich in den der Aufblähung des Fortsatzes über. Peripher verliert sie sich mebr oder weniger schnell in den wieder schmalen Fortsatz. Manchmal bemerkt man noch eine zweite, leichtere Erweiterung desselben Fortsatzes. Die Zellen selbst, deren Axenzylinderfortsätze diese isolierten Schwellungen aufweisen, sind gewöhnlich weniger aufgebläht. Der Kern liegt auch öfters weniger exzentrisch.

$\mathrm{Ob}$ das geschilderte Verhalten dieser Fortsätze bei allen Pyramidenzellen. der Rinde vorkommt, lässt sich schwer behaupten. Man sieht es in allen untersuchten Windungen (Frontal-, vordere und hintere Zentral-, Calcarina-Windungen) und meistens in der Schicht der kleinen und mittelgrossen Pyramiden. Bei ətwas dickeren Schnitten (15 $\mu$ wie sie Fig. 1 , und 4 darstellen), bei möglichst radiärer Schnittfäche und auf stärker gefärbten Präparaten kommen diese Aufblähungen sehr zahlreich zum Vorschein. Oft sieht man auch Gebilde, die der Form und Struktur nach ihnen entsprechen, aber olne zugehörigen Zellkörper wahrscheinlich Abschnitte von in einem anderen Schnitt liegenden Zellen bilden (Fig. 4b). Die schwache Färbbarkeit erschwert die Untersuchung dieser Gebilde.

Die Seitendendriten der Zellen sind ziemlich weit verfolgbar und meistens breiter als normal. Auch an ihnen begegnet man - aber sehr selten - kleinen Aufblähungen. Oefters trifft man nur Erweiterungen an den Dichotomiestellen. Es ist möglich, dass ähnliche Auftreibungen wie am Axenzylinderfortsatz, sich zahlreicher auch an den Dendriten befinden, aber der rasche Zerfall der von der Zelle mehr entfernten Teile der Dendriten in einzelne Bröckel macht die Zugehörigkeit solcher Gebilde zu Dendriten unsicher.

DerApikalfortsatz ist meistens gleichmässig erweitert, färbt sich ziemlich weit und zeigt oft die wabjge Struktur der Zelle. Einzelne Nissl Schollen kommen hier aber öfters zum Vorschein.

Im Kleinhirn zeigen die Purkinjeschen Zellen denselben Anteil an der allgemeinen Schwellung, die aber hier meistens lokalisiert ist, während der andere Teil der Zelle mit dem verlagerten Kern ziemlich normale Verhält-. nisse mit konzentrisch angeordneten Nissl-Schollen aufweist. Die Den- 
driten sind sehr blass gefärbt und besonders am Anfang und an den Dichotomiestellen stark erweitert. Die Struktur der aufgeblähten Teile ist dieselbe netzartige, wie wir sie in den Zellen der Grosshirnrinde beobachtet haben.

Sehr viele Purkinjeschen Zellen müssen ganz verloren gegangen sein, da deren Zahl kaum mehr als $1 / 4$ bis $1 / 6$ der normal auf gleichem Raum vorhandenen beträgt. Die Molekularschicht des Kleinhirns ist sehr erheblich verschmälert, ziemlich genau auf die Hälfte ihres Durohmessers. An der Körnerschicht der Kleinhirnwindungen sind keine abnormen Befunde wahrgenommen.

Soweit das Toluidinblau- oder Methylenblau-Präparat einen Einblick in das Verhalten der Gliazellen und der Gefässe erlaubt, ist darüber folgendes zu bemerken:

Die Zahl der Gliaelemente ist etwas grösser als auf gleicher Fläche am normalen Gehirn zu erwarten. Dies dürfte dadurch bedingt sein, dass in dem extrem atrophischen Gehirn die einzelnen Elemente näher zusammenrücken. Die Gliaelemente selbst weisen meistens leichte Degenerationszeichen auf. Man sieht viele kleine, im ganzen sehr dunkelblau gefärbte Kerne, die dreieckig oder hantelförmig geschrumptt sind. Ihr Protoplasmahof kommt in gekörnten und geschlängelten Zügen zum Vorschein, was am besten in der plexiformen Schicht zu bemerken ist. Hier trifft man - sehr seiten - einige Gebilde, deren Protoplasma rasenartig zusammenfliesst. - Die grösseren, hellblau gefärbten Kerne sind sehr oft viereckig oder unregelmässig verunstaltet und enthalten einige kleine dunkle Körner an der Innenwand der Membran. Der sie umgebende Protoplasmaleib zeigt manchmal eine oder ein paar Vacuolen im Innern, oder bildet ringförmige, aus Körnern bestehende Züge.

Sehr schön kann man das Verhalten der Gliaelemente zu den Ganglienzellen beobachten. Ausser gewöhnlichen Trabantkörnern sieht man an vielen Stellen, wie 3-5 Gliakerne von verschiedenen Seiten der Zelle bis zur Hälfte in ihrer Wand liegen. An anderen Orten findet man 1-3 Gliakerne ganz im Leibe einer schwer erkrankten Zelle.

An den Gefässen der Rinde sieht man keine gröberen Veränderungen. Hier und da ist ihr Verlauf etwas geschlängelt, die Gefässwand etwas gefaltet und an einigen Stellen seheint eine Einlagerung von hyaliner Substanz zu beginnen. An den Gefässwandelementen ist nichts abnormes wahrzunehmen.

Was uns die nach Kulschitzki-Wolters gefürbten Präparate der Rinde bieten, lässt sich in kurzen Worten sagen:

Die Radiärfaserung und das intertadiäre Flechtwerk ist in allen Windungen ziemlich gut erhalten; am besten in Zentral- und Kalkarinarinde, am stärksten gelichtet in Frontal- und Temporalwindungen. Auffallend schlecht färben sich im ganzen die Schnitte aus dem linken Temporallappen. Das superradiäre Flechtwerk und die tangentiale Randzone ist in allen Gegenden stark reduziert; am wenigen noch in den Zentralwindungen. Der Gennarische Streifen ist am besten in der Kalkarinarinde erhalten, 
aber auch hier stark gelichtet. In anderen Windungen ist er meistens nur durch einzelne horizontale Fasern markiert.

Ich möchte aber diesen Befunden nicht zu viel Gewicht beilegen, da das Gehirn schon seit 1901 in Kalium bichromicum lag. Andererseits hatte ich kein normales Material entsprechenden Alters zum Vergleichen.

Auf wenig differenzierten Präparaten kommen auch an dem chrom. gehärteten Gehirn die geschwollenen Zellen deutlich zum Vorschein. Sie sind durch ein Häufchen kleiner graver Körner um den dunklen, an der Seite liegenden Kern dargestellt. Sogar die Aufblähung des Basalfortsatzes ist durch diese Körner angedeutet.

Dasselbe Vorkommen kanu man noch besser auf den Eisenhaematoxilin-Präparaten nach Heidenhain beobachten. Je nach der kürzeren oder längeren Differenzierung der Präparate ist der Kern im Ganzen dunkel oder nur sein Chromatingerüst und das dunkle Kernkörperchen gefärbt. Das Protoplasma der Zelle und der aufgeblähte Nervenfortsatz zeigen auf diffusem, mehr oder weniger stark grau gefärbtem Hintergrunde verschieden grosse dunkle Körner. Von den Dendriten kommt nur der Apikaldendrit zum Vorschein.

Die bald nach dem Tode untersuchten Sehnerven nach WeigertPal-Färbung zeigten keinen Faserausfall.

Fassen wir jetzt kurz die wichtigsten Merkmale des geschilderten Falles zusammen, wie er sich uns klinisch und anatomo-pathologisch darstellt:

Christliche Abstammung, keine Belastung, keine Familiarität. Wie ibre Geschwister soll sich auch unsere Kranke bis zum 7. Jahre körperlich und geistig normal entwickelt haben. Während aber alle anderen gesund wurden, blieb sie ohne sichtlichen Grund geistig zurück. (Den überstandenen Krankheiten könnte man vielleicht nur sekundär disponierende Ursache zuschreiben.) Im genauer nicht zu bestimmenden Alter, jedenfalls vor dem 10. Lebensjahre, beginnt die Sehschärfe abzunehmen; man diagnostiziert Atrophia nervi optici und die beschriebene ejgentümliche Makulaaffektion mit Pigmenteinwanderung in die Retina. Gegen das 13. Jahr gesellen sich Störung an Sprache und Gang dazu, ein Jahr später treten epileptische Anfälle und dann auch Nystagmus auf. Alle diese Krankheitssymptome nehmen in chronischer, jahrelang dauernder Entwicklung zu, bis die Kranke blind, verblödet, spastisch gelähmt und stumm geworden ist. In den letzten Lebensjahren tritt Kräfteverfall hinzu und mit 26 Jahren stirbt sie im höchsten Marasmus.

Die Sektion der inneren Organe zeigt uns nur die letzte Ursache des Todes und die durch Inanition verursachten Veränderungen. Was wir an dem makroskopischen Befunde des Gehirns und der Meningen 
finden, gibt uns auch keine Anhaltspunkte für die Erklärung des Krankheitsprozesses. Es fällt nur das geringe Gehirngewicht von $670 \mathrm{~g}$ bei $51 \mathrm{~cm}$ Schädelumfang und 17:15 $\mathrm{cm}$ Schädeldurchmesser auf.

Lassen die Krankengeschichte und der makroskopische Befund die Diagnose unsicher, so gibt uns die mikroskopische Untersuchung Aufschluss über die Art der Erkrankung. Der elektive Schwellungs prozess der Ganglienzellen der Rinde aller Gehirnlappen und aller Schichten1), bei den nur geringfügigen sekundären Veränderungen anderer Bestandteile des Gehirns, lässt im Zusammenhang mit den klinischen Symptomen und mit der Entwicklung der Krankbeit keinen Zweifel übrig, dass wir es hier mit der juvenilen Form der amaurotischen Idiotie zu tun haben. Ob der Erkrankungsprozess sich auch auf die Ganglienzellen des Rückenmarkes erstreckte und dadurch ubiquitär war, wie es die Sicherheit der Diagnose erfordert, kann ich nicht sagen, da mir leider nur das früher erwähnte Material zur Verfügung stand. Es scheint mir aber, dass durch diesen Umstand die Diagnose nicht zweifelhafter wird.

Es bleibt uns noch übrig, die für den untersuchten Fall gegenüber den sonst beschriebenen Fällen eigenen Merkmale des klinischen und anatomischen Bildes hervorzuheben.

Das erste, was dem Falle eine Sonderstellung gibt, ist das Fehlen der Familiarität. Von vier Kindern ist nur unsere Kranke an diesem Prozesse erkrankt.

Weiter verdient der Augenhintergrund ein paar Worte Besprechung.

Für die typischen Fälle der Tay-Sachsschen Krankheit (infantiler Form der amaurotisch-familiären Idiotie), die vor oder kurz nach dem zweiten Lebensjahre starben, ist die bekannte Makulaveränderung charakteristisch, wie sie zuerst noch 1881 Waren-Tay ${ }^{2}$ ) selbst beschrieben hat: "In beiden Augen war in der Gegend des gelben Fleckes ein auffälliger, ziemlich begrenzter, grosser weisser Fleck, mehr oder weniger rund, der im Zentrum einen braunroten, ziemlich runden Punkt zeigte, der stark mit dem weissen Fleck, der ihn umgab, kontrastierte." $\mathrm{Zu}$ dieser Makulaaffektion gesellt sich meistens etwas später Atrophie der Papille. Nur bei einem kleinen Prozentsatz der beschriebenen Fälle von infantiler Form wurde diese Veränderung der Makula vermisst.

1) Nur ein Teil der tieferen Pyramiden in den Zentralwindungen zeigt sklerotische Veränderungen.

2) Waren-Tay, Symmetrical changes in the region of the yellow-spot in each eye of an infant. - Transactions of the ophtalmological society of the united Kingdom 1881; - zit. nach Falkenheim: Ueber familiäre amaurotische Idiotie. - Jahrb. f. Kinderheilk., 1901. 
Von den länger lebenden Fällen des infantilen Typus begegnen wir bei dem J. Köller'schen Fall $\left.{ }^{1}\right)$ (1900 acht Jahre alt) neben dem typischen Befund an der Makula, scharf begrenzten, atrophischen, gelblich verfärbten Papillen, wie in Fällen von Retinitis pigmentosa. - Der 1. Fall von Mülberger ${ }^{2}$ ) zeigte im Alter von $3 \frac{1}{4}$ Jahren in der Makulagegend neine blassrötliche Fläche von einem annähernd kreisrunden, schollenartig angeordneten Pigmentsaum von schwarzer Farbe eingeschlossen". Bei einem 2. Fall $-11^{1 / 4}$ Jahre alt - sehen wir "in der Makulagegend keine gröberen Veränderungen, und nahe der Peripherie schmale, gezackte Pigmentzüge von schwarzer Farbe" ${ }^{3}$ ).

Was die juvenile Form dieser Erkrankung anbetrifft, so weisen die ophthalmoskopisch untersuchten Fälle von Higier ${ }^{4}$ ) und Vogt5) nur Atrophie der Papille ohne Veränderung der Makulagegend auf. In einem Falle von Vogt (Fall III, 1) zeigt der Ophthalmoskop ausserdem einige unregelmässige pigmentierte Stellen. - Bei allen vier Geschwistern von Spielmeyer ${ }^{6}$ ) haben wir es mit einer bestimmten Retinitis pigmentosa zu tun. Beim zweiten Kind sind wenige Pigmenteinlagerungen auch am hinteren Pol vorhanden; beim vierten können nur einige Pigmenteinlagerungen in der äussersten Peripherie der Netzhaut festgestellt werden.

Stock ${ }^{7}$ ) der diese Fälle untersucht hatte, sagt dazu: „Dass hier der Degenerationsprozess der Retina schon am weitesten zurückliegt, das sieht man einmal an der Schrumpfung der Retina und dann an ihrer Pigmentierung. Dass diese Pigmentierung nicht als ein integrie-

1) Koller, Two cases of "a rare fatal disease of infancy, with symmetrical changes in the macula lutea" (Kingdom). New York medical Record, 1896; zitiert nach Falkenheim.

2) A. Mülberger, Die familiäre amaurotische Idiotie und ihre Diagnose. Münch. med. Wochensehr., 1903, Nr. 45.

3) Herr A. Mülberger war so freundlieh, mir folgendes mitzuteilen: "Trotz der von meiner Seite aus gemachten Anstrengungen gelang es damals nicht, die Erlaubnis zur Sektion der beiden Kinder zu bekommen. Die beiden Kinder sind bald nach meiner Publikation, d. h. das ältere $1 / 2 \mathrm{~J}$, das jüngere $11 / 4 \mathrm{~J}$. nachher gestorben."

4) L. c. -1896.

5) L. c. -1905 .

6) L. c. -1907.

7) Stock, Ueber eine besondere Form der familiären amaurotischen Idiotie. - Bericht der 23. Versamml. d. Ophthalm.-Ges. Heidelberg 1906. Wiesbaden, 1907; zitiert nach Vogt. 
render Bestandteil zu unserer Erkrankung gehört, sieht man daran, dass sie in einem Falle (IV. Fall) so gut wie fehlt."

Bei unserer Kranken finden wir, wie der erst erhobene Befund lautet: „Papillen blass und undurchsichtig. Makula in ganz bestimmter Weise erkrankt: um eine hellrote ovale Figur geht ein dichter gefranster Pigmentkranz herum, und dies auf beiden Augen ganz gleich". Prof. $\mathrm{Haab}^{1}$ ) macht dazu folgende Bemerkung: „Ob diese Nakulaerkrankung sich aus der Tay-Sachsschen Form entwickelte, ist unsicher, aber nicht unmöglich. Jedenfalls war da eine ältere Erkrankung vorhanden." Die nach $3 / 4$ Jahren vorgenommene Untersuchung zeigt, dass die Einwanderung des Pigments in die Retina Fortschritte macht, denn man sieht , in der Peripherie, schon ziemlich bald gegen dieselbe beginnend, feine bräunliche Tüpfelung auf graurotem Grund ..."

Zwischen den Fällen der amaurotischen Idiotie scheinen mir diese durch Pigmenteinwanderung ausgezeichneten Fälle nicht ohne Interesse zu sein. Sie könnten vielleicht - besonders der I. Fall von Mülberger und unsere Kranke, bei welchen sekundäre Pigmenteinlagerung. in der Gegend der Makula stattfindet - einen Uebergang zwischen beiden Typen der amaurotischen Idiotie darstellen. Ich meine insofern, dass vielleicht bei ihnen in einem nicht zur Untersuchung gelangten Stadium eine Makulaveränderung stattfand, die mehr dem typischen Befunde ähnlich war und erst später durch sekundäre Veränderungen verwischt wurde. Wenn es so wäre - die Frage muss jetzt offen bleiben so fiele der bis jetzt wichtigste Streitpunkt weg, ob die Fälle von juveniler Form trotz des Fehlens der typischen Makulaaffektion zur TaySachsschen Krankbeit gerechnet werden dürfen oder nicht. Denn, was den anatomischen Befund am Zentralnervensystem anbetrifft, weisen die beiden Formen nur quantitative, dem mehr oder weniger resistenteren Gewebe entsprechende Unterschiede auf.

Auch in dieser Richtung bieten die am Gehirn unserer Kranken erhobenen Befunde gewisses Interesse:

Wenn ich meine Methylen- oder Toluidinblaupräparate mit der Beschreibung der Veränderungen der Ganglienzellen von Hirsch und mit den Abbildungen auf dieselbe Weise gefärbten Präparate von Schaffer einerseits, von Spielmeyer und Vogt andererseits vergleiche, so komme ich ungezwungen zu dem Schluss, dass Art und Schwere der Zellveränderungen bei unserer Kranken sich viel mehr den Veränderungen bei infantiler Form nähern, als es bei anderen Fällen von juvenilem Typus gefunden wurde.

1) Laut der privaten brieflichen Mitteilung. 
Bei seinen genauen Untersuchungen erwähnt schon Hirsch${ }^{1}$ ), dass die geschwollenen Zellen ein feines Retikulum enthalten. Schaffer ${ }^{2}$ ) charakterisiert dann ausführlicher die Art der Erkrankung folgendermassen: "Ueberblicken wir das zytopathologische Nissl-Bild, so lässt sich, kurz gefasst, sagen, dass sich hier ebenso wie an den Fibrillenbildern eine Schwellung des Zellkörpers und den Dendriten zeigt. Im Verlauf der Zellschwellung wird der Zellkern exzentrisch gelegen, dann erleidet das Tigroid eine progressive Verkleinerung bis zum völligen Schwund ohne jedwelche Spur des Zerfalls, wodurch das spongioplasmatische Netzwerk, welches normaliter durch das Tigroid förmlich verhïllt ist, in deutlicher Form sichtbar wird; parallel geht die Aufblähung des plasmatischen Netzwerkes, wodurch in charakteristischer Weise engere perinukleäre und klaffendere periphere Netzlücken entstehen. In der Reihenfolge später erfolgt die Desintegration des Spongioplasmas". Austerdem hebt Schaffer noch die Schwellung der Dendriten, wodurch ihre netzförmige Struktur zum Vorschein kommt, hervor.

Vergleichen wir nun die Beschreibung von Schaffer mit unseren Präparaten, so sehen wir, dass die Art der Erkrankung der Ganglienzellen in beiden Fällen fast übereinstimmt; die beigegebenen Figuren gleichen fast ganz den von Schaffer ${ }^{3}$ ) gegebenen Abbildungen. Der Unterschied, der sonst besteht, ist nur graduell: Die Sehwellung der Zellen in unserem Fall ist meistens nicht so gross, die Auftreibung der Zellfortsätze ist bedeutend weniger ausgesprochen. Es fehlt weiter die Desintegration des Spongioplasmas bei schwer erkrankten Zellen. Nirgends kann man eine diffus gefärbte Staubmasse, die aus dem zerfallenen Netzwerk entsteht, finden. Sogar in sehr stark aufgeblähten Zellen (Fig. 6) kann man auf dem gleichmässig gefärbten Hintergrunde der Aufblähung doch ein feines Retikulum bemerken. Weiter begegnet man hier in den Zentralwindungen den erwähnten sklerotischen Veränderungen der Ganglienzellen. Vielleicht gehen auch hier nicht alle geschwollenen Zellen auf diese Weise zu Grunde, dass die Wände der peripheren Maschen bersten, sondern dass sie in sklerotische Formen übergehen.

1) W. Hirsch, The pathological anatomy of a fatal disease of infancy with symmetrical changes in the yellow spot (Waren-Tay). Yournal of Nervous and Mental Diseases. 1898. Zitiert nach Schaffer.

2) L. c. 1909 .

3) K. Schaffer, Ueber die Pathohistologie eines neuen Falles (VIII.) von Sachsscher familiär-amaurotischer Idiotie usw. Journal f. Psych. u. Neurolog. Bd. X. 1909. - Fig. 21, 22, 26. 
Was die Nisslschen Schollen anbelangt, so bemerkt man von ibnen fast gar nichts. Die Knotenpunkte des Protoplasmanetzes sind nur sehr schwach durch dunklere Färbung und Verdickung markiert. Die chromatische Substanz wird wahrscheinlich durch den Erkrankungsprozess mehr oder weniger schnell in eine von anderen mikrochemischen Eigenschaften umgewandelt. Dass sie, wie es Schaffer vermutet, allmählich aufgebracht würde, lässt sich, scheint mir, nicht sagen. Wir wissen von ihr nur so viel, dass sie bei dieser Erkrankung im Nisslschen Aequivalentbilde nicht mehr zum Vorschein kommt.

Noch einen Punkt möchte ich hervorheben: Sich auf seine Fibrillenpräparate stützend betont Schaffer ausdrücklich in seiner letzten Arbeit ${ }^{1}$ ), dass gegenüber der Aufschwellang der Zellen und der Dendriten der Axenzylinderfortsatz (Nervenfortsatz) vollkommen intakt, frei von Erkrankung bleibt, und er zieht daraus gewisse Schlüsse auf die pathologische Bedeutung dieser Erscheinung. Wenn man dagegen seine Zellbilder ansieht, so bemerkt man, dass neben der nicht so häufigen und weniger starken Aufblähung der anderen Dendriten, vor allem der mittlere, radiär verlaufende Basalfortsatz der Pyramidenzellen an der isolierten Anschwellung leidet. Schaffer selbst hebt es mit folgenden Worten hervor: „Ich möchte die morphologisch nicht uninteressante Tatsache hervorbeben, dass die keulen- und sackförmige Auftreibung überwiegend an den Basaldendriten, hingegen am Apikaldendrit nur selten anzutreffen ist."

Ich konnte zwar die Hirnrinde unserer Kranken nicht auf Fibrillenpräparaten untersuchen, man bemerkt aber auch schon auf den Toluidinblaupräparaten dieselbe bäufige Lokalisation der Aufblähung. Nur Schaffers Erklärung von diesem Befunde scheint mir unverständlich zu sein. Der Umstand, dass es sich meistens um mittlere, selten um seitliche Basalfortsätze handelt, die einen radiären Verlauf zeigen, dass man an ihnen keine dichotomischen Verzweigungen, und gegenüber den anderen Zellfortsätzen nur sehr wenig oder gar nichts ron chromatischer Substanz sieht, spricht gerade aller Wahrscheinlichkeit nach dafür, dass die betroffenen Fortsätze Axenzylinderfortsätze darstellen, die nicht nur mit anderen Zellfortsätzen an dem Schwellungsprozesse teilnehmen, sondern demselben viel häufiger und schwerer unterliegen.

Nach all den Ausführungen sehe ich mich berechtigt zum folgenden Schlusse mit bezug auf den von mir beschriebenen Fall:

Klinisch zeigt er alle Merkmale der juvenilen Form der amau-

1) L. c. 1909 . 
rotischen Idiotie. Anatomisch - soweit das Material die Untersuchung erlaubte - steht er dem infantilen Typus dieser Erkrankung sehr nahe. Der Unterschied, der besteht, ist einerseits durch den chronischen Verlauf bedingt; andererseits dadurch, dass der Erkrankungsprozess nicht junges Nervengewebe von den ersten Lebensmonaten trifft, sondern älteres von grösserer Widerstandsfähigkeit.

Rheinau 1909.

\section{Erklärung der Abbildungen (Tafel XVIII).}

Fig. 1. Durchschnitt einer Frontalwindung; es fehlt nur unten die Schicht der Spindelzellen. Mit Leitz Obj. 5, Occ. 1 gezeichnet. Methylenblaufärbung.

Fig. 2. Gesichtsfeld aus der Schicht der mittelgrossen Pyramidenzellen einer Frontalwindung. Leitz Obj. 5, Occ. 1. Mikrophotographie, Haemalaunfärbung. a) Gewebslücken; b) Auftreibung des Basalfortsatzes.

Fig. 3. Verschiedene Ganglienzellen aus der vorderen Zentralwindung. Mit Leitz Oel-Immersion 1/12, Occ. 1 gezeichnet. Toluidinblaufärbung. a) Kleine obere Pyramidenzelle; b) Spindelzelle; c) d) Polymorphe Zellen aus der oberen Sohicht.

Fig. 4. Gesichtsfeld aus der Schicht der mittelgrossen Pyramidenzellen der vorderen Zentralwindung. a) Eine fast ganz zerfallene Zelle; b) Abschnitt einer Zelle oder eines aufgeblähten Basalfortsatzes.

Fig. 5. Gesichtsfeld aus der Schicht der kleineren Pyramidenzellen der vorderen Zentralwindung. Ausser der deutlich zum Vorschein kommenden netzartigen Struktur der geschwellten Zellen sieht man bei a) die aufgetriebenen Basalfortsätze. (Mikrophotographie.)

Fig. 6. Zwei unregelmässig, stark aufgeblähte, grosse Pyramidenzellen. a) Aus der Frontalwindung; $b$ ) aus der Zentralwindung.

Vergrösserung und Färbung bei Fig. 4, 5 und 6 wie bei Fig. 3 . 


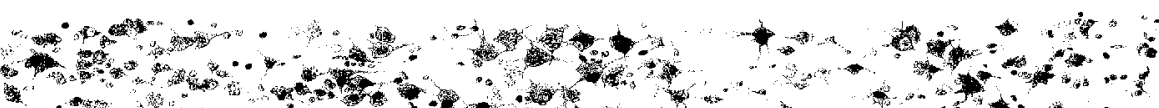

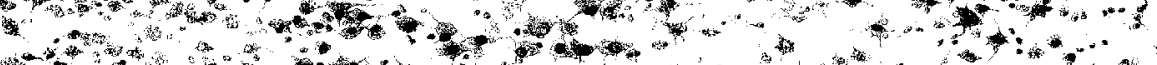
* a

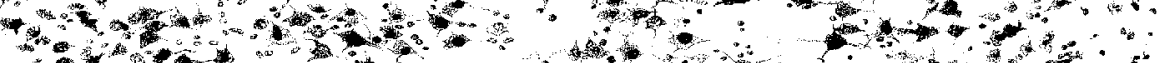

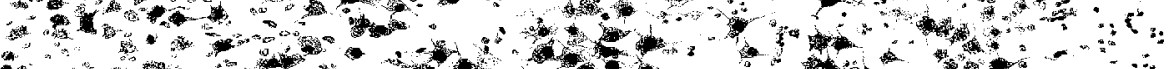

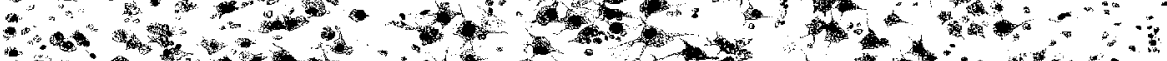
by

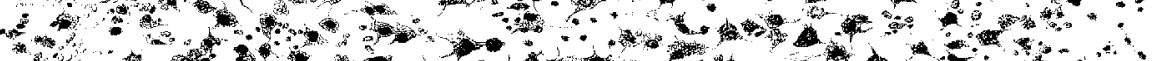

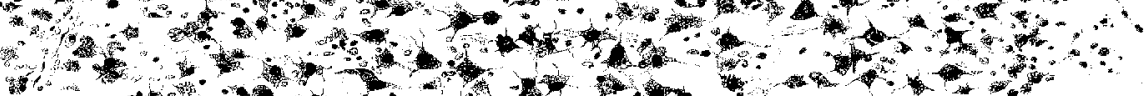

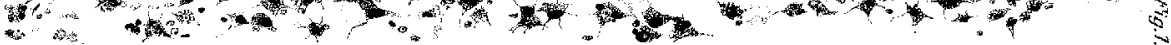

(

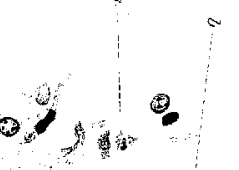
$\%$

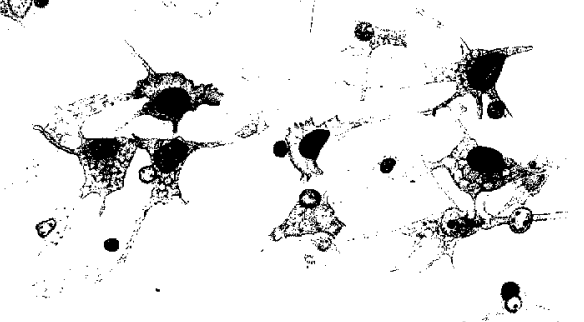

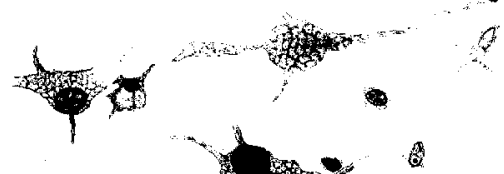<smiles>[CH]1[CH]C=C1</smiles>
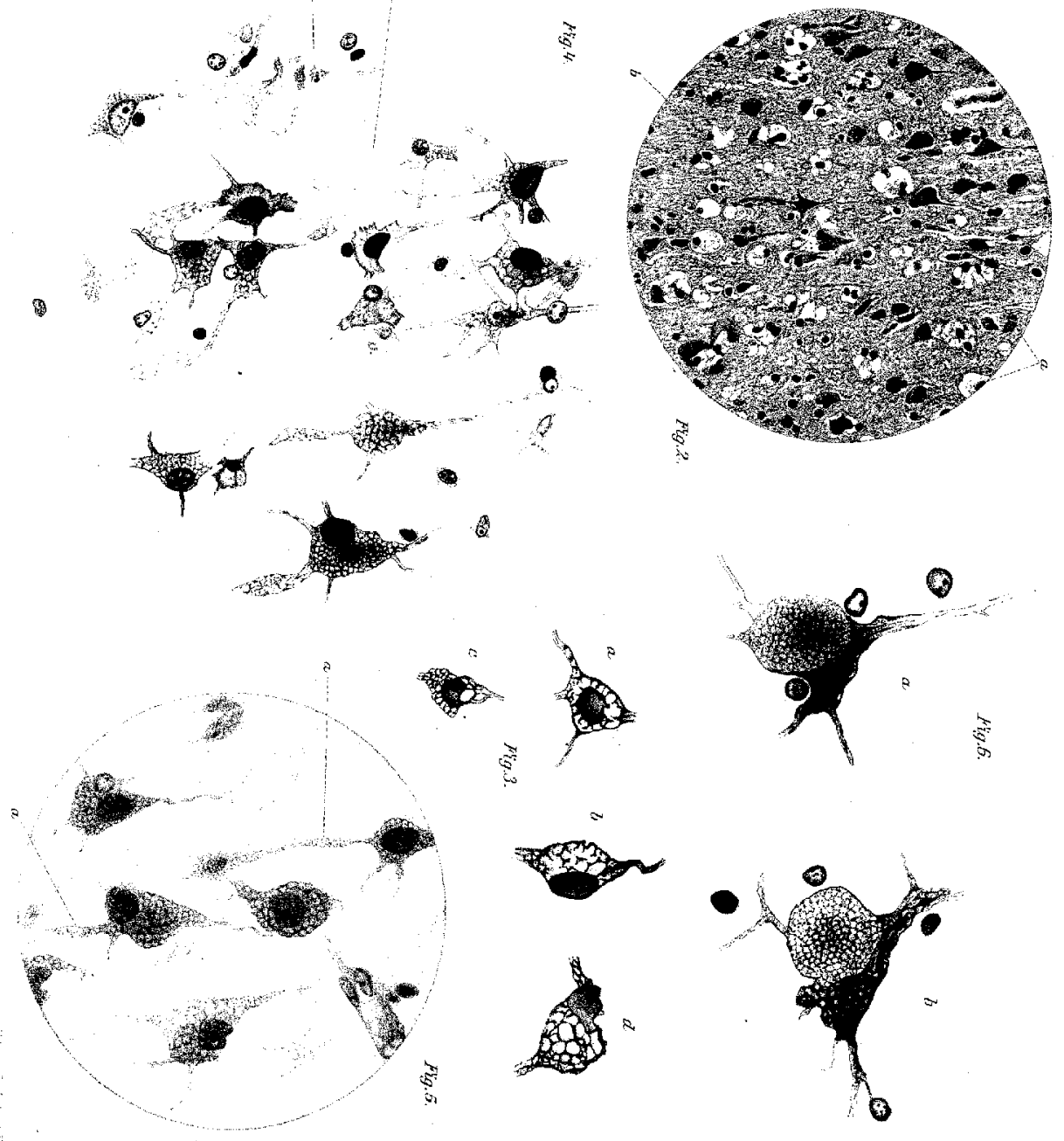<smiles></smiles>

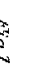

